\title{
A Simple Soft Limiter Describing Function for Biomedical Applications
}

\author{
Annraoi de Paor and John Ringwood*, Senior Member, IEEE
}

\begin{abstract}
This paper suggests an arctangent function as a suitable parameterisation for the soft-limiting gain characteristic frequently encountered in models of biomedical systems. This function is shown, as an example, to fit the neural arc component of the baroreflex with the main contribution of the paper being the development of a simple describing function (DF) characteristic for the arctangent. The simple form of the DF allows transparency of the physiological parameters in, for example, stability analysis. For illustration, the derived DF is used to examine low-frequency limit cycles in blood pressure, sometimes termed Mayer waves.
\end{abstract}

Index Terms-Baroreflex, describing function, limit cycle.

\section{INTRODUCTION}

A LL real systems are characterized by finite limits on system variables, such as temperatures, pressures, concentrations, displacements, etc. In man-made systems, this is usually represented by a hard saturation characteristic which observes the upper and lower bound on a variable. Physiological systems, however, are generally not as "mechanical" and are usually characterized by soft limiting functions. In addition to soft-limiting characteristics, sigmoidal characteristics have been observed in a variety of physiological control systems, such as neuron recruitment [1] and the blood pressure regulation (baroreflex) system [2].

A number of physiological subsystems [3], such as blood pressure control [4] and the tubuloglomerular feedback system [5], exhibit sustained oscillations which are thought to be limit cycles, i.e., they are a consequence of the feedback system themselves. While oscillations in many physiological variables are deliberate and easily explained, such as the cardiac rhythm in blood pressure, the reason for oscillation in the above cited examples is not so clear. Indeed, some suggest that the parameters of such oscillations could form the basis for diagnostic measures, since the presence/absence, amplitude and frequency of oscillations can sometimes be related to physiological/pathological condition, for example haemorrhage [6]. In general, for stable limit cycles to develop, the feedback loop must contain some nonlinear element and sigmoid-like nonlinearities have been modelled in both the baroreflex (relay [7] and sigmoid [8])

Manuscript received April 20, 2005; revised December 18, 2005. This work was supported in part by the Wellcome Trust. Asterisk indicates corresponding author.

A. de Paor is with the Department of Electronic and Electrical Engineering, the National University of Ireland at Dublin, Belfield, Dublin 4, Ireland

*J. Ringwood is with the Department of Electronic Engineering, the National University of Ireland at Maynooth, County Kildare, Ireland (e-mail: john.ringwood@eeng.nuim.ie).

Digital Object Identifier 10.1109/TBME.2006.873681

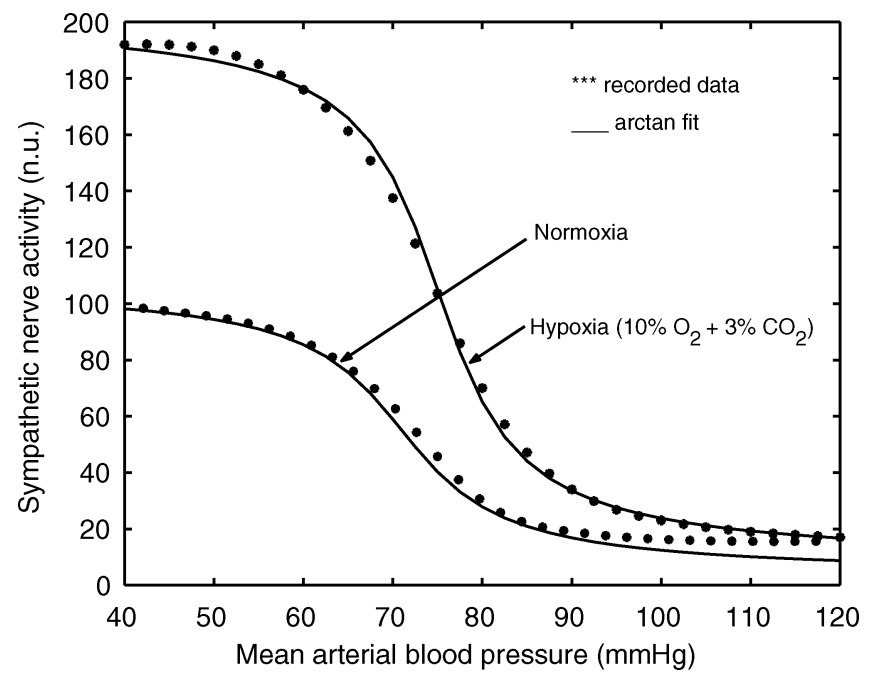

Fig. 1. Arctan function fits to baroreflex data.

and tubuloglomerular (hyperbolic tangent) cases. Indeed, variations in the sigmoid shape have been observed during changes in physiological condition (see Fig. 1 for a hypoxia case) and these shape changes can be used to predict changes in oscillation condition using relatively straightforward analysis [9], [10]. However, if measurement of such oscillations can provide diagnostic information about the physiology, there must be the following:

1) an analysis route connecting the physiological parameters to those of the oscillation;

2) transparency of the physiological parameters in the oscillation parameters.

Condition 1) above is relatively straightforward, via the describing function (DF) technique [9] and this has been used in the physiology setting to examine low-frequency (circa. $0.1 \mathrm{~Hz}$ in humans) oscillations in blood pressure [8]. However, achieving 2) has proved somewhat elusive, with highly unwieldy DF expressions resulting from a variety of approximations [11], [12] to the sigmoid characteristics, necessitated due to the inability to get closed-form DF expressions for the original sigmoid descriptions.

This paper proposes the ubiquitous arctangent function as a suitable parameterisation for sigmoidal characteristics typical in physiological subsystems. It is shown how a DF description for the arctan function can be developed without the need for any approximation to the function and, most importantly, the final developed analytical connections between the oscillation parameters (such as amplitude) and the physiology are simple, allowing clear connections to be made between possible pathologies and measured oscillation parameters. 
The remainder of the paper is organized as follows: Section II examines the validity of using an arctan function to approximate sigmoidal curves typically found in physiological application and compares the arctan description to others typically employed. Section III provides the essence of the paper in developing the DF for the arctan function, while Section IV provides a brief analysis confirming the monotonicity of the derived DF. Section V looks at how the DF can be employed in limit cycle analysis and example calculations using the DF are given in Section VI. A short discussion on simplifications used in the paper is given in Section VII and, finally, conclusions are drawn in Section VIII.

\section{SigmoidAl CURVES}

A variety of sigmoid-like parameterisations, both symmetric and asymmetric, are reported in the literature, many of which are reported in [10]. These include the following.

- The symmetrical sigmoid, employed by Ringwood and Malpas [8]

$$
s_{1}(x)=\frac{h}{1+e^{-\beta\left(x-x^{*}\right)}}-\frac{h}{1+e^{\beta\left(x-x^{*}\right)}}+y^{*}
$$

where

$x \quad$ input to the characteristic;

$s_{i}(x)$ output of characteristic $i$;

$\beta \quad$ "curvature" (input scaling) parameter;

$h \quad$ is the vertical range of the characteristic;

with the characteristic centered at $\left(x^{*}, y^{*}\right)$.

- The alternative symmetrical sigmoid, used by Head and McCarthy [13], Kingwell et al. [14], and others

$$
s_{2}(x)=h+\left(\frac{y^{*}+2 h}{1+e^{\beta\left(x-x^{*}\right)}}\right)+y^{*} .
$$

- The hyperbolic tangent, used by Seidel and Herzel [15] and Eyal and Akselrod [16]

$$
\begin{aligned}
s_{3}(x) & =h\left(\tanh \left(\beta\left(x-x^{*}\right)\right)\right)+y^{*} \\
& =h\left(\frac{e^{\beta\left(x-x^{*}\right)}-e^{-\beta\left(x-x^{*}\right)}}{e^{\beta\left(x-x^{*}\right)}+e^{-\beta\left(x-x^{*}\right)}}\right)+y^{*} .
\end{aligned}
$$

- The asymmetric sigmoid function of Ricketts and Head [17]

$$
s_{4}(x)=\frac{2 h}{1+f(x) e^{-\beta_{t}\left(x-x^{*}\right)}+(1-f(x)) e^{-\beta_{b}\left(x-x^{*}\right)}}+h+y^{*}
$$

where

$$
f(x)=\frac{1}{1+e^{\bar{c}\left(x-x^{*}\right)}} \quad \text { and } \quad \bar{c}=\frac{2 \beta_{t} \beta_{b}}{\left|\beta_{t}+\beta_{b}\right|}
$$

where $\beta_{t}$ and $\beta_{b}$ describe the curvature at top and bottom of the curve individually, respectively.
It is debatable as to the value of an asymmetric curve, though a degree of asymmetry can be observed in the cardiac baroreflex [18].

Reviewing the range of sigmoid descriptions in (1) to (6), a common feature is the presence of an exponential term in the input, $x$. This, aside from any other complications, leads to difficulties in analytical evaluation of the Fourier integrals associated with DF calculations for these characteristics and has necessitated the polynomial approximations used in [11] and [12]. One sigmoid description which avoids the use of exponential terms is the Hill function, as used by Abbiw-Jackson and Langford [19]

$$
s_{5}(x)=h\left(\frac{\left(x-x^{*}\right)^{n}}{\left(a-x^{*}\right)^{n}+\left(x-x^{*}\right)^{n}}\right)+y^{*} .
$$

Though this function has no exponential terms, an analytic solution to the Fourier integral cannot be found with all of $n, a$, $x^{*}$, and $y^{*}$ specified as variables, though solutions can be found for fixed $n$ over specific ranges of $a$. In addition the following hold.

- The Hill function is naturally asymmetric and can be difficult to make symmetric over all ranges.

- Some criteria developed by Heidel and Moloney [20] suggest that the Hill function, developed primarily for applications in pharmacology, is not a good candidate for the baroreflex curve data, following application to a range of test points on the curve.

Our proposal is to use the arctangent function, of the form

$$
s_{a}(x)=h \tan ^{-1}\left(\beta\left(x-x^{*}\right)\right)+y^{*} .
$$

This function provides a simple, symmetrical description of a sigmoid-like function and can, without approximation, be manipulated in a DF calculation to yield an attractive simple result. Note that the output range (upper limit to lower limit) of the arctan function is $h \pi$, from (8).

The applicability of the arctan function in fitting (at least some) physiological data is confirmed by Fig. 1, which shows the fit achieved to the (vasoresistive) baroreflex data of Malpas [2] for normoxia (control) and hypoxic conditions, with parameters as documented in Table I. This fit was achieved using manual manipulation of the arctan parameters (indicative of the transparent relationship between the function parameters and its appearance), but a suitable optimisation routine (which minimises the fit error) could also have been easily employed.

Note that the negative values for $\beta$ give the 'reversed' sigmoid appearance. This form, as modelled in the baroreflex, results from the use of the curve as a control element following negative feedback (i.e., the input, $x$, is effectively negated).

\section{DF DEVELOPMENT}

The DF technique [9] assumes a (single frequency) sinusoidal input to the nonlinear element, $f(x)$, of the form

$$
x(t)=M \sin \theta, \quad \theta=\omega_{o} t .
$$


TABLE I

ARCTAN PARAMETERS FOR BAROREFLEX CURVES

\begin{tabular}{|l|c|c|c|c|}
\hline & $h$ & $\beta$ & $x^{*}$ & $y^{*}$ \\
\hline Normoxia & 33 & -0.12 & -71 & 55 \\
Hypoxia & 62 & -0.15 & -75 & 105 \\
\hline
\end{tabular}

The output is then expressed in a Fourier series, as

$$
y(t)=a_{o}+\sum_{i=1}^{\infty} a_{i} \sin (i \theta)+b_{i} \cos (i \theta)
$$

with

$$
a_{i}=\frac{1}{\pi} \int_{-\pi}^{\pi} f(x) \sin (i \theta) d \theta, \quad b_{i}=\frac{1}{\pi} \int_{-\pi}^{\pi} f(x) \cos (i \theta) d \theta .
$$

For brevity, during the DF calculation, we will assume that $x^{*}=$ $y^{*}=0$ in (8), with the corollary that the input sinusoid will be centered on the sigmoid. Preserving this situation, vertical and horizontal translation of the function can easily be added at a later stage by suitable choice of $x^{*}$ and $y^{*}$. Thus, for the moment $\left(x^{*}=y^{*}=0\right), a_{o}=0$ and for single valued symmetric functions, it can easily be shown that all $b_{i}$ terms are zero. Also, the $a_{i}$ terms can be evaluated over $0 \rightarrow \pi$ or $0 \rightarrow(\pi / 2)$ as desired. The DF, representing the gain between the fundamental of the output and the input, can now be defined as

$$
\mathrm{DF}(M)=\frac{a_{1} \sin \theta}{M \sin \theta}=\frac{a_{1}}{M} .
$$

For the sigmoid of (8), with $x^{*}=y^{*}=0$

$$
\mathrm{DF}(M)=\frac{2 h}{\pi M} \int_{0}^{\pi} \tan ^{-1}(\beta M \sin \theta) \sin \theta d \theta
$$

Equation (13) can be recast as

$$
\mathrm{DF}(M)=\frac{2 h}{\pi M} \int_{0}^{\pi} \tan ^{-1}(\beta M \sin \theta) \frac{d}{d \theta}(\cos \theta) d \theta
$$

and integrated, by parts, to give

$$
\begin{aligned}
\operatorname{DF}(M) & =\frac{2 h}{\pi M} \int_{0}^{\pi} \cos \theta \frac{d}{d \theta}\left(\tan ^{-1}(\beta M \sin \theta)\right) d \theta \\
& =\frac{2 h \beta}{\pi} \int_{0}^{\pi} \frac{\cos ^{2} \theta}{1+\beta^{2} M^{2} \sin ^{2} \theta} d \theta
\end{aligned}
$$

with the second term of the "integration by parts" disappearing at both upper and lower limits. Equation (16) is easily recast as

$$
\begin{aligned}
\mathrm{DF}(M)= & \frac{2 h \beta}{\pi} \int_{0}^{\pi} \frac{1}{1+\beta^{2} M^{2} \sin ^{2} \theta} d \theta \\
& -\frac{2 h}{\pi \beta M^{2}} \int_{0}^{\pi} \frac{\beta^{2} M^{2} \sin ^{2} \theta}{1+\beta^{2} M^{2} \sin ^{2} \theta} d \theta \\
= & \frac{2 h \beta}{\pi} \int_{0}^{\pi} \frac{1}{1+\beta^{2} M^{2} \sin ^{2} \theta} d \theta \\
& -\frac{2 h}{\pi \beta M^{2}} \int_{0}^{\pi}\left(1-\frac{1}{1+\beta^{2} M^{2} \sin ^{2} \theta}\right) d \theta \\
= & \frac{2 h \beta}{\pi}\left(1+\frac{1}{\beta^{2} M^{2}}\right) \int_{0}^{\pi} \frac{d \theta}{1+\beta^{2} M^{2} \sin ^{2} \theta} \\
& -\frac{2 h}{\beta M^{2}} .
\end{aligned}
$$

The remaining integral in (17) may be evaluated [21] as

$\int_{0}^{\pi} \frac{d \theta}{1+\beta^{2} M^{2} \sin ^{2} \theta}=\frac{1}{\sqrt{1+\beta^{2} M^{2}}} \tan ^{-1}\left(\sqrt{1+\beta^{2} M^{2}} \tan \theta\right)$.

Though the integral appears to be zero at both limits, observation of the symmetry of the integrand in (17) allows the integral to be recast as

$$
\int_{0}^{\pi} \frac{d \theta}{1+\beta^{2} M^{2} \sin ^{2} \theta}=2 \int_{0}^{\frac{\pi}{2}} \frac{d \theta}{1+\beta^{2} M^{2} \sin ^{2} \theta}
$$

and, with the final observation that

$$
\lim _{\theta \rightarrow \frac{\pi}{2}} \tan ^{-1}(P \tan \theta)=\frac{\pi}{2} \forall P
$$

the DF in (17) may be evaluated as

$$
\mathrm{DF}(M)=\frac{2 h}{\beta M^{2}}\left(\sqrt{1+\beta^{2} M^{2}}-1\right) .
$$

\section{A. Asymptote for Large $M$}

The asymptotic approximation of the DF for $M \rightarrow \infty$ is easily obtained from (21) as

$$
\mathrm{DF}(M) \rightarrow \frac{2 h}{M}, \quad M \rightarrow \infty .
$$


This is easily verified intuitively, due to the saturation effect of the $\tan ^{-1}$ function.

\section{B. Asymptote for Small $M$}

Using the first two terms of a Maclaurin series, the integrand in (17) may be approximated (for small $M$ ) as

$$
\frac{1}{1+\beta^{2} M^{2} \sin ^{2} \theta} \approx 1-\beta^{2} M^{2} \sin ^{2} \theta, \quad M \ll 1 .
$$

Using this in (17) gives

$$
\begin{aligned}
\operatorname{DF}(M)= & \frac{2 h \beta}{\pi}\left(1+\frac{1}{\beta^{2} M^{2}}\right) \\
& \times\left(\pi-\beta^{2} M^{2} \int_{0}^{\pi} \frac{1-\cos 2 \theta}{2} d \theta\right)-\frac{2 h}{\beta M^{2}} \\
= & \frac{2 h \beta}{\pi}\left(1+\frac{1}{\beta^{2} M^{2}}\right) \pi\left(1-\frac{\beta^{2} M^{2}}{2}\right)-\frac{2 h}{\beta M^{2}} \\
= & h \beta\left(1-\beta^{2} M^{2}\right) .
\end{aligned}
$$

The expression in (24) can be used to determine the zero-amplitude limit of the DF and is used in the stability analysis of Section V-C.

\section{Monotonicity OF DF}

An important characteristic of the DF is whether it is monotonic in $M$, or not, i.e., whether $\mathrm{DF}(M)$ increases or decreases consistently with increasing $M$. This has consequences for the stability analysis in Section $\mathrm{V}$ in terms of a unique solution for the limit cycle amplitude. The DFs of previous approximations have displayed a variety of properties: the polynomial approximation of Kinnane et al. [12] is monotonic, while the Taylor series approximation of Holohan [11] is not.

Using the DF expression in (21), the monotonicity of this function may be determined by examining the derivative (or slope) of the DF with respect to $M$, i.e., if the slope of $d(D F) / d M$ is always positive (or negative), then the DF is monotonic. Evaluating the derivative

$$
\frac{d(D F)}{d M}=\frac{\frac{2 \beta^{3} M^{3} h}{\sqrt{1+\beta^{2} M^{2}}}-4 h \beta M\left(\sqrt{1+\beta^{2} M^{2}}-1\right)}{\beta^{2} M^{4}} .
$$

Clearly, the denominator (always positive) does not affect the sign of $d(D F) / d M$ and it is sufficient to focus on the sign of the numerator. Multiplying across numerator terms by $\sqrt{1+\beta^{2} M^{2}}$ (always positive) and collecting terms reduces to

$$
-2-\beta^{2} M^{2}+2 \sqrt{1+\beta^{2} M^{2}}
$$

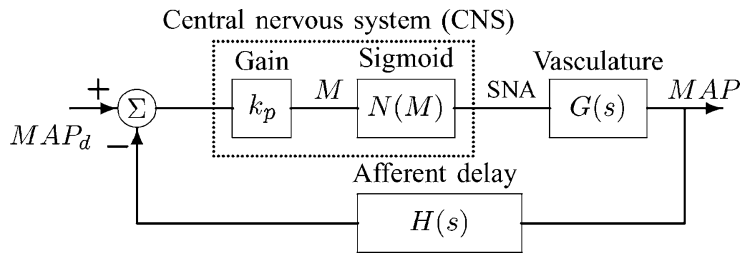

Fig. 2. Baroreflex feedback loop.

or, with appropriate scaling

$$
2-\frac{1}{\sqrt{1+\beta^{2} M^{2}}}-\sqrt{1+\beta^{2} M^{2}}
$$

Now, since

$$
\frac{1}{a}+a>2 \quad \forall \quad a>0
$$

we get that

$$
2-\frac{1}{\sqrt{1+\beta^{2} M^{2}}}-\sqrt{1+\beta^{2} M^{2}}<0 \quad \forall \quad M
$$

and we can conclude that the DF expression in (21) decreases monotonically for increasing $M$.

\section{OSCILLATION EXAMPLE}

\section{A. Nonlinear Feedback Model}

An example of the peripheral resistance (PR) ${ }^{1}$ baroreflex in the rabbit, similar to the model documented in [8], will be used here to illustrate the use of the DF in stability and diagnostic assessment. The essential components of the PR baroreflex model are shown in Fig. 2, consisting of delays due to nerve conduction time (in $G(s)$ and $H(s)$ ), operation of the controlling action of the central nervous system (represented by $k_{p}$ and the sigmoid, $N(M)$ ) and the dynamics of the vasculature (in $G(s)$ ), with:

$$
G(s)=\frac{e^{-s \tau_{e}}}{1+s \tau_{v}}, \quad H(s)=e^{-s \tau_{a}} .
$$

Note the folowing:

- $\tau_{a}$ represents afferent (both preganglionic and postganglionic) nerve delay;

- $\tau_{e}$ represents efferent nerve delay;

- the dynamic lag is the vasculature, $\tau_{v}$, is primarily due to the dynamics of contraction of the smooth muscle surrounding the arterioles;

- for convenience, the gain term, $k_{p}$, will be absorbed into the input scaling term, $\beta$, of the arctan function as

$$
\beta^{*}=\beta k_{p}
$$

${ }^{1}$ Note that the full baroreflex comprises both PR and cardiac output subsystems. 
TABLE II

Model Parameter VALUes

\begin{tabular}{|c|c|c|c|}
\hline$k_{p}$ & $\tau_{e}$ & $\tau_{a}$ & $\tau_{v}$ \\
\hline 3.0 & 0.67 & 0.2 & 10.0 \\
\hline
\end{tabular}

Note also that the steady-state (dc) gain of $G(s)$ has been normalized. This is partly due to the fact that there is some ambiguity over the dc gain between efferent sympathetic nerve activity (SNA) and mean arterial pressure (MAP) and partly due to the fact that such gain has been absorbed elsewhere (in $k_{p}$ and $N(M)$ ). The sigmoidal nonlinearity, $N(M)$, can be represented as in Fig. 1, with the "normalized units" (NU) again showing the ambiguity over SNA quantification. This ambiguity is due to the fact that current SNA sensors do not recruit a consistent (or measurable) number of nerve fibers. The model, via the values in Table II, is parameterized for the rabbit [22], with the value of $k_{p}$ chosen to reflect the experimental observation that no oscillation in MAP exists in the nominal (control) situation shown in Fig. 1, while a distinct oscillation at about $0.3 \mathrm{~Hz}$ exists in the case of hypoxia. Note that the oscillation frequency, and model parameters, are species dependent [8]. For this parameterisation, the feedback loop oscillates with a frequency of $0.2967 \mathrm{~Hz}$, for the hypoxia case only.

\section{B. Validity of DF Approximation}

From Section III, it is seen that the DF is essentially an input/ output gain representation at a fundamental frequency. The assumption that the input is of single frequency and the discarding of all higher harmonics can now be examined in a physiological setting.

Most, but not all, naturally occurring systems respond better to low frequencies (i.e., are "low-pass") rather then higher frequencies. An example of this is the renal vasculature, as quantified by [23] and Guild et al. [24]. It can be seen that the block representing the dynamics of the vasculature in Fig. 2 contains a first order low-pass element, giving a relative reduction of 6 $\mathrm{dB}$ (factor of 2) at $0.6 \mathrm{~Hz}$, compared to $0.3 \mathrm{~Hz}$. The more comprehensive dynamical model of Guild et al. [24] gives a relative gain reduction of $12 \mathrm{~dB}$ (factor of 4). While these quantities are not excessively large, they are sufficient to significantly reduce the loop gain at harmonic frequencies to a value below that required for sustained oscillations, and it is reasonable to conclude that the second (and higher) harmonics play no part in the sustained oscillations and a description which only models the fundamental (i.e., the DF) is sufficient. Spectra of blood pressure [22] also demonstrate no harmonics of the low-frequency oscillation.

Note that some researchers [25] have included a mild highpass dynamical block to represent the baroreceptors. However, this does not produce any significant effect at the frequency of interest.

\section{Stability Assessment}

Following the analysis route described in [8], we can examine for sustained limit cycle oscillations by looking for an intersection between the $G H(j \omega)$ curve and the $-1 / \mathrm{DF}(M)$ line in the

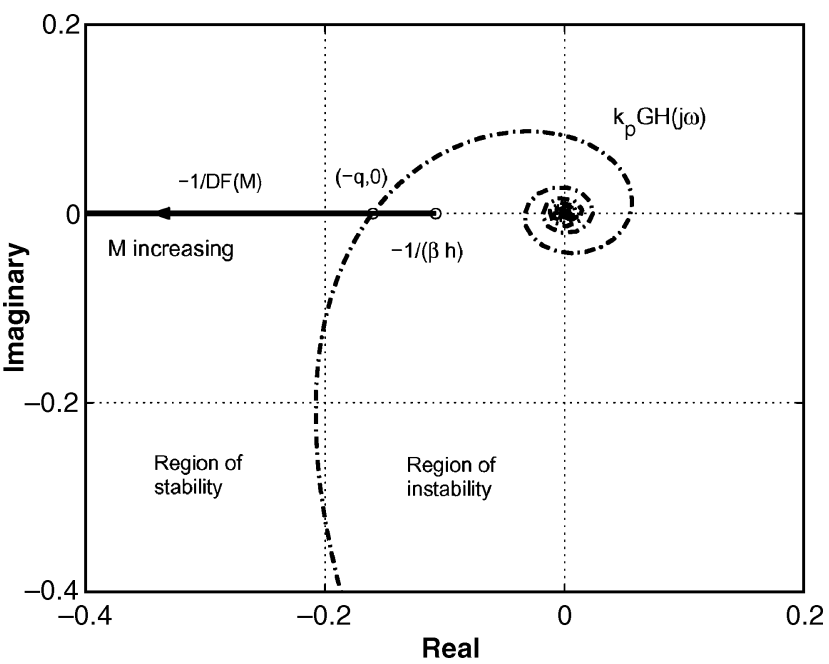

Fig. 3. Stability diagram for nonlinear feedback loop.

complex plane. This is illustrated in Fig. 3. Intersection between the $G H(j \omega)$ curve and the $-1 / \operatorname{DF}(M)$ line achieves a stable limit cycle, since operation in the 'region of instability' causes an increase in $M$ and movement towards $(-q, 0)$, while operation in the 'region of stability' causes a decrease in $M$, also resulting in movement towards $(-q, 0)$. Note that the rightmost limit of the $-1 / \mathrm{DF}(M)$ is given by

$$
-\frac{1}{D F(0)}=\frac{1}{h \beta^{*}}
$$

using the "small $M$ " asymptote developed in (24).

Since $G H(j \omega)$ is a function of frequency, $\omega$, and $-1 / \operatorname{DF}(M)$ is a function of amplitude, $M$, we can solve for the limit cycle amplitude and frequency at the intersection point. Denoting $(-q, 0)$ as the intersection point, we can solve for limit cycle amplitude and frequency, respectively, as

$$
\frac{1}{q}=D F\left(M_{o}\right)=\frac{2 h}{\beta^{*} M_{o}^{2}}\left(\sqrt{1+\beta^{* 2} M_{o}^{2}}-1\right)
$$

and

$$
-\omega_{o} \tau_{e}-\omega_{o} \tau_{a}-\tan ^{-1}\left(\omega_{o} \tau_{v}\right)=-\pi .
$$

Note that, since $-1 / \operatorname{DF}(M)$ is always real, the intersection is always achieved on the negative real axis. Therefore, the procedure to determine $\omega_{o}$ and $M_{o}$ may be stated as follows.

1) Solve (34) for $\omega_{o}$.

2) Determine $q$ from $\left|G H\left(j \omega_{o}\right)\right|$, or

$$
q=\frac{1}{\sqrt{\omega_{o}^{2} \tau_{v}^{2}+1}} .
$$

3) Solve for $M_{o}$ from (33).

Though (34) is nonlinear and does not have an exact analytical solution, we can observe that the following approximation is 
valid for the range of parameter and frequency values typical in baroreflex oscillations: $\left(10<\omega_{o} \tau_{v}<30\right)$ :

$$
\tan ^{-1}(x) \approx \frac{\pi}{2}-\frac{1}{x}, \quad x \gg 1
$$

reducing (34) to the quadratic

$$
\tau_{v}\left(\tau_{e}+\tau_{a}\right) \omega_{o}^{2}-\frac{\pi}{2} \tau_{v} \omega_{o}-1=0
$$

with solution

$$
\omega_{o}^{1,2}=\frac{\frac{\pi}{2} \tau_{v} \pm \sqrt{\frac{\pi^{2}}{4} \tau_{v}^{2}+4 \tau_{v}\left(\tau_{e}+\tau_{a}\right)}}{2 \tau_{v}\left(\tau_{e}+\tau_{a}\right)} .
$$

Since

$$
\frac{\pi}{2} \tau_{v}<\sqrt{\frac{\pi^{2}}{4} \tau_{v}^{2}+4 \tau_{v}\left(\tau_{e}+\tau_{a}\right)} \quad \forall \quad \tau_{v}, \tau_{e}, \tau_{a}>0
$$

only one positive solution for $\omega_{o}$ is permitted as

$$
\omega_{o}=\frac{\frac{\pi}{2} \tau_{v}+\sqrt{\frac{\pi^{2}}{4} \tau_{v}^{2}+4 \tau_{v}\left(\tau_{e}+\tau_{a}\right)}}{2 \tau_{v}\left(\tau_{e}+\tau_{a}\right)} .
$$

The validity of the approximation in (36) (and, therefore, the approximate solution in (40)) is enhanced by noting that the product $\omega_{o} \tau_{v}$ is relatively invariant across species, i.e., for smaller species (e.g., rats), $\omega_{o}$ increases, while $\tau_{v}$ decreases, with the converse being true for larger species (e.g., humans).

The uniqueness of a solution to (33) is guaranteed since the monotonicity of $\mathrm{DF}(M)$ (and hence $-1 / \mathrm{DF}(M)$ ) was proven in Section IV. This is not, for example, the case with the sigmoid DF approximation in [11], where the $-1 / \mathrm{DF}(M)$ line doubles back on itself.

Finally, a solution to (33) for $M_{o}$ may be achieved by proceeding as follows. From (33)

$$
\beta^{*} M_{o}^{2}=2 h q\left(\sqrt{1+\beta^{* 2} M_{o}^{2}}-1\right)
$$

or

$$
\sqrt{1+\beta^{* 2} M_{o}^{2}}=\frac{\beta^{*} M_{o}^{2}}{2 h q}+1 .
$$

Squaring both sides and dividing across by $\beta^{*} M_{o}^{2}$ gives

$$
1=\frac{M_{o}^{2}}{4 h^{2} q^{2}}+\frac{1}{\beta^{*} h q}
$$

yielding a solution for $M_{o}$ as

$$
M_{o}=2 h q \sqrt{1-\frac{1}{\beta^{*} h q}} .
$$

\section{EXAMPLE CALCULATIONS}

Some sample calculations will now be shown to illustrate the use of the DF. The case taken will be that for the rabbit parameters (detailed in Section V-A) for the normoxia and hypoxia cases, detailed in Section II.

\section{A. Normoxia}

Following the procedure in Section $\mathrm{V}-\mathrm{C}$, we first determine the (first) frequency for which the imaginary part of $G H(j \omega)$ is zero. Using (40), this can be determined as $\omega_{o}=1.86702$ rads/s [exact frequency response gives a value of 1.86707 , justifying the approximation in (36)]. This is equivalent to 0.2972 $\mathrm{Hz}$, comparing well to the value obtained from simulation in Section V-A. The magnitude (equal to the negative of the real part, in this case) of $G H(j \omega)$ is easily evaluated from (35) as $q=0.05 \dot{3}$. However, checking the value for the rightmost limit of $-1 / \operatorname{DF}(M)$ gives a value of $-1 /\left(\beta^{*} h\right)=-0.084$ and clearly there is no intersection of the $G H(j \omega)$ and $-1 / \mathrm{DF}(M)$ loci. Therefore, we conclude that there is no oscillation for this case.

\section{B. Hypoxia}

For the hypoxia case, there is significantly more "gain" in the baroreflex curve (see Fig. 1) and an intersection exists between the $G H(j \omega)$ and $-1 / \operatorname{DF}(M)$ loci. Again, using the value of $q=0.05 \dot{3}$ (from Section VI-A), the amplitude of the limit cycle oscillation can now be determined from (44) as

$$
M_{o}=2 h q \sqrt{1-\frac{1}{\beta^{*} h q}}=3.78 \mathrm{mmHg}
$$

which is confirmed by simulation (using the MATLAB SIMULINK environment) and, qualitatively, typical of experimental observations [2], [22].

\section{DISCUSSION}

While the approach outlined in this paper avoids approximation of the function which provides the basis for the DF, some milder simplifications and approximations have been made to achieve transparency in the result and these are summarized in the following text.

The DF, as detailed in Section III [see (12)], assumes an input sinusoid of a single frequency. The nonlinear soft limiter characteristic will, however, produce harmonics of this single frequency which may propagate around the feedback loop (Fig. 2). The attenuation of these harmonics has been dealt with in Section V-B and previous work in this area [26] has also confirmed 
that the first harmonic at the nonlinearity output is approximately $30 \mathrm{~dB}$ down on the fundamental, corresponding to a reduction factor of 0.03 . Therefore, it is reasonable to assume that the single frequency DF is valid in this case.

Another approximation which is introduced is that in (36). A typical value for $\omega_{o} \tau_{v}$ (e.g., in the example used) is 18.67 , resulting in values for the left- and right-hand sides of (36) of $\tan ^{-1}(x)=1.51728$ and $(\pi / 2)-(1 / x)=1.51723$, respectively, confirming the validity of the approximation. Section VI-A also confirms that the solution for $\omega_{o}$ is correct to 5 significant places.

Finally, the "small-amplitude" asymptote for the DF, as used in the stability analysis of Section V-C to evaluate $D F(0)$, involves an approximation. As a reference, the exact DF of (21) can be used to determine the value for $D F(0)$ using l'Hopital's rule (since $\lim _{M \rightarrow 0} \mathrm{DF}(M)$ is indeterminate), giving an identical result to (24) of $h \beta$. This is not surprising, since (23) becomes exact at $M=0$.

\section{CONCLUSION}

This paper develops a DF for a sigmoid-type characteristic found in a variety of physiological subsystems. The DF permits an analysis (e.g., stability analysis) to be performed on predominantly linear systems containing such a nonlinear element. The relatively simple form of the DF in (21) leads to a transparency between, for example, oscillation parameters (in the PR baroreflex case) and contributing physiological parameters. For example, it is clear from the solution for the oscillation amplitude, $M_{o}$, in (44) that oscillation amplitude increases as follows:

- with increase in $h$ and, less directly, with increase in $\beta^{*}$ (due to an increase in $\beta$ or $k_{p}$ ), which represent the output and input "gains" of the sigmoid, respectively;

- with increase in $q$, resulting from an increase in gain of the vasculature dynamics (normalized to unity in the example shown).

Note also that $q$ could also increase with an increase in the pure delay (phase) of the system (resulting in clockwise rotation of the $G H(j \omega)$ curve). However, this physiological parameter is unlikely to change due to pathology or intervention.

The position of the right-hand extreme of the $-1 / \operatorname{DF}(M)$ curve, relative to the value of $q$, determines whether oscillations will be present or absent. The onset of oscillations is determined when $1 / \beta^{*} h$ (determined from (24) with $M=0$ ) becomes equal to $q$. Therefore, the likelihood of oscillations also increases with increases in $\beta, k_{p}, h$, and $q$. Note that the condition for onset of oscillations can also be found by examining for the point where the solution for $M_{o}$ [in (44)] becomes imaginary. This occurs when $\left(1-\left(1 / \beta^{*} h q\right)\right)=0$ or $\left(1 / \beta^{*} h\right)=q$.

In addition to the simple, transparent, solution for the DF, one of the chief merits of the DF calculation in this paper is that it is based on the sigmoid itself, rather then an approximation to it. The approximation of Holohan [11] is inaccurate for any values of $M$ marginally greater then zero, while the approximation of Kinnane et al. [12] penalises error uniformly across the range of $M$, resulting in regions of poor approximation, particularly for small $M$.

\section{ACKNOWLEDGMENT}

The authors are grateful for the contribution to this work by Prof. S. Malpas of the University of Auckland and Dr. O. Kinnane of NUI Maynooth.

\section{REFERENCES}

[1] A. Branner, R. B. Stein, and R. A. Normann, "Selective stimulation of cat siatic nerve using an array of varying-length microelectrodes," $J$. Neurophysiol., vol. 85, no. 4, pp. 1585-1594, Apr. 2001.

[2] S. C. Malpas, R. D. Bendle, G. A. Head, and J. H. Ricketts, "Frequency and amplitude of sympathetic discharges by baroreflexes during hypoxia in conscious rabbits," Am. J. Physiol.: Heart Circ. Physiol., vol. 271, no. 6, pp. H2563-H2574, 1996.

[3] H. M. Power, "Marvellous control systems of the heart," Electron. Power, vol. 21, pp. 183-188, Feb. 1975.

[4] A. M. Malliani, F. Pagani, F. Lombardi, and S. Cerutti, "Cardiovascular neural regulation explored in the frequency domain," Circ. Res., vol. 84, pp. 482-492, 1991.

[5] H. E. Layton, E. B. Pitman, and L. C. Moore, "Spectral properties of the tubuloglomerular feedback system," Am J. Physiol.: Renal Physiol., vol. 273, pp. F635-F649, 1997.

[6] S. C. Malpas and D. E. Burgess, "Renal SNA as the primary mediator of slow oscillations in blood pressure during haemorrhage," Am. J. Physiol.: Heart Circ. Physiol., vol. 279, no. 3, pp. H1299-H1306, 2000.

[7] R. I. Kitney, "A nonlinear model for studying oscillation in the blood pressure control system,” J. Biomed. Eng., vol. 1, pp. 89-99, 1979.

[8] J. V. Ringwood and S. C. Malpas, "Slow oscillations in blood pressure via a nonlinear model," Am. J. Physiol.: Comp., Regul. Int. Physiol., vol. 280, no. 4, pp. R1105-R1115, 2001

[9] D. P. Atherton, Nonlinear Control Engineering. New York: Van Nostrand Reinhold, 1982.

[10] O. P. Kinnane, "Investigation of slow oscillations in blood pressure," Ph.D. thesis, National Univ. Ireland, Maynooth, 2005.

[11] A. M. Holohan, "On calculating the describing function," in Proc. Irish Signal and Systems Conf., Dublin, Ireland, 2000, pp. 466-470.

[12] O. P. Kinnane, J. V. Ringwood, D. Kelly, and S. C. Malpas, "Describing function approximation for biomedical engineering applications," in Proc. Irish Signal and Systems Conf., Belfast, Ireland, 2004, pp. 107-112.

[13] G. Head and R. McCarthy, "Vagal and sympathetic components of the heart rate range and gain of the baroreceptor-heart rate reflex in conscious rats," J. Autonom. Nervous Syst., vol. 21, pp. 203-213, 1987.

[14] B. A. Kingwell, G. A. McPherson, and P. I. Korner, "Assessment of gain of tachycardia and bradycardia responses of cardiac baroreflex," Am. J. Physiol.: Heart Circ. Physiol., vol. 260, pp. H1254-H1263, 1991.

[15] H. Seidel and H. Herzel, "Bifurcations in a nonlinear model of the baroreceptor-cardiac reflex," Physica D, vol. 115, pp. 145-160, 1998.

[16] S. Eyal and S. Akselrod, "Bifurcations in a simple model of the cardiovascular system," Meth. Inf. Med., vol. 39, pp. 118-121, 2000.

[17] J. H. Ricketts and G. A. Head, "A five-parameter logistic equation for investigating asymmetry of curvature in baroreflex studies," Am. J. Physiol.: Comp., Regular and Integrated Physiol., vol. 46, pp. R441-R454, 1999.

[18] G. A. Head, "Cardiac baroreflexes and hypertension," Clin. Exp. Pharmacol. Physiol., vol. 21, pp. 791-802, 1994.

[19] R. M. Abbiw-Jackson and W. F. Langford, "Gain-induced oscillations in blood pressure," J. Math. Biol., vol. 37, pp. 203-234, 1998.

[20] J. Heidel and J. Maloney, "When can sigmoidal data be fit to a Hill curve?," J. Austral. Math Soc. Ser. B, vol. 40, pp. 1-10, 1998.

[21] K. Rektorys, "Entry No. 279," in Survey of Applicable Mathematics. London, U.K.: Iliffe, 1964.

[22] S. C. Malpas, "Neural influences on cardiovascular variability: possibilities and pitfalls," Am. J. Physiol.: Heart Circ. Physiol., vol. 282, pp. H6-H20, 2002.

[23] D. E. Burgess, J. D. Hundley, S.-G. Li, D. C. Randall, and D. R. Brown, "First-order differential-delay equation for the baroreflex predicts the 0.4-Hz blood pressure rhythm in rats," Am. J. Physiol.: Comp., Regul. Int. Physiol., vol. 273, pp. R894-R903, 2001.

[24] S.-J. Guild, P. C. Austin, M. Navakatikyan, J. V. Ringwood, and S C. Malpas, "Dynamic relationship between sympathetic nerve activity and renal blood flow: a frequency domain approach," Am. J. Physiol.: Comp., Regul. Int. Physiol., vol. 281, pp. R206-R212, 2001. 
[25] T. Kawada, C. Zheng, Y. Yanagiya, K. Uemura, T. Miyamoto, M. Inagaki, T. Shishido, M. Sugimachi, and S. Sunagawa, "High-cut characteristics of the baroreflex neural arc preserve baroreflex gain against pulsatile pressure," Am. J. Physiol.: Heart Circ. Physiol., vol. 282, pp. H1149-H1156, 2002.

[26] J. V. Ringwood and O. P. Kinnane, "Prediction of low frequency blood pressure oscillations via a combined heart/resistance model," in Proc. 16th IFAC World Congr., Prague, Czech Republic, Jul. 2005.

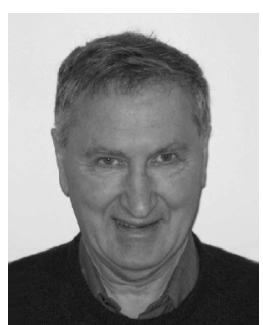

Annraoi de Paor was born in Waterford, Ireland, on August 5, 1940. He received the B.E., Ph.D., and D.Sc. degrees in electrical engineering from the National University of Ireland, Dublin, in 1961, 1967, and 1974, respectively. He received the M.S. degree in Electrical Engineering from the University of California at Berkeley in 1963.

He lectured at the University of Salford, Salford, , U.K., from 1963 to 1967, and the National University of Ireland, Dublin, from 1967 to 1969. In 1969, he was appointed Professor of Control Engineering at the University of Salford and on January 1, 1978 took up his current position as Professor of Electrical Engineering at the National University of Ireland, Dublin. He has published in control theory, biomedical engineering, renewable energy systems, classical electrical engineering, geomagnetism, and engineering education. Since 1976, he has been a regular visitor at Universities in Slovakia.

Dr. de Paor is a member of the Royal Irish Academy and a fellow of the Institution of Engineers of Ireland, the Institution of Electrical Engineers, and the Institute of Mathematics and its Applications.

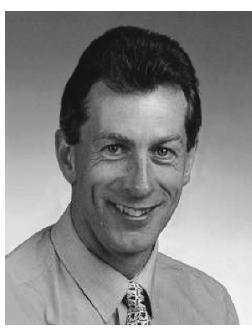

John Ringwood received the Diploma degree in electrical engineering from Dublin Institute of Technology, Dublin, Ireland, and the Ph.D. degree in control systems from Strathclyde University, Strathclyde, U.K., in 1981 and 1985, respectively.

He was with the School of Electronic Engineering, Dublin City University, frpm 1985 to 2000 and during that time held visiting positions at Massey University and the University of Auckland in New Zealand. He is currently Professor of Electronic Engineering with the National University of Ireland (NUI), Maynooth, and is Dean of the Faculty of Engineering. He was Head of the Electronic Engineering Department at NUI Maynooth from 2000 until 2005, developing the Department from a greenfield site. He has acted as consultant to a number of companies, in the power, servomechanism and process industries. His interests cover a number of areas, including time series modeling, control of wave energy systems, control of plasma processes, biomedical engineering and e-learning.

Dr. Ringwood is a Chartered Engineer and a fellow of both the Institute of Electrical Engineers and the Institution of Engineers of Ireland. 